\title{
Serum cortisol level as a useful predictor of surgical disease in patients with acute abdominal pain
}

\author{
OZGUR DIKME 1 OZLEM DIKME
}

1 Emergency Department, Istanbul Training and Research Hospital, University of Health Sciences, Istanbul, Turkey

2 Emergency Department, Koc University, Istanbul, Turkey

Corresponding author:

Ozgur Dikme

Kasap Ilyas mh.

Org. Nafiz Gurman cd. Istanbul Egitim ve Arastirma Hastanesi Acil Servisi

Fatih, Istanbul. Postal code: 34098

Phone: +905053511602

Fax: +902124596000

E-mail:drozgurdikme@yahoo.com

\section{ABSTRACT}

Introduction. The immediate aim should be to identify potentially resuscitative acute abdominal pain that requires prompt investigation or early surgical intervention. We aimed to evaluate whether serum cortisol levels differentiate surgical and non-surgical disease in patients with acute abdominal pain.

Materials and methods. In this prospective cross-sectional study, the primary end-point was defined as differences in serum cortisol levels between surgically and non-surgically treated patients with non-traumatic acute abdominal pain. The secondary end-point was to compare the cortisol levels with defined complete blood count (CBC) parameters in those groups.

Results. One hundred eleven patients with acute abdominal pain were included in the study. Three most frequent diagnoses were nonspecific abdominal pain, acute appendicitis and dyspeptic complaints. Thirty patients were hospitalized and 22 of them were operated. The median cortisol level was $23.13 \mu \mathrm{g} / \mathrm{dl}$ in surgically treated patients and $13.94 \mu \mathrm{g} / \mathrm{dl}$ in non-surgically treated patients $(\mathrm{p}<0.001)$. The area under the ROC curve using cortisol to detect surgical disease was 0.750 (95\% CI, 0.6590.827 ) and the accuracy of cortisol to detect surgical disease was not inferior to defined CBC parameters. A cortisol value of $17.98 \mu \mathrm{g} / \mathrm{dl}$ had a sensitivity of $67.4 \%$ and a specificity of $77.3 \%$ for surgical disease. Conclusion. Operated patients had higher serum cortisol levels. High serum cortisol levels may indicate surgical disease at the early stage on admission in ED patients with acute abdominal pain and may be used as a marker in the prediction of acute surgical abdomen.
Key words: abdominal pain, cortisol, emergency department, surgery

\section{INTRODUCTION}

Abdominal pain is one of the most common admission complaints at the emergency departments (EDs). Pain of less than one week's duration that may require urgent surgery is defined as acute abdominal pain. Abdominal pain is a condition that almost everyone has encountered at least once in their lifetime, and has a broad differential diagnosis, including benign and life-threatening diseases. In 2009, $8 \%$ of all ED patients in the United States (10.41 million patients) presented with abdominal pain. (1) The variability of symptoms and clinical features of many serious causes of abdominal pain has led to doctors relying heavily on diagnostic tests. As reported in a ED based study, $65 \%$ of patients with undifferentiated non-traumatic abdominal pain received at least one diagnostic test during their evaluation. (2) Patients with acute abdominal pain are a heterogeneous group that consumes a great deal of ED's resources. The immediate aim should be to identify potentially resuscitative acute abdominal pain that requires prompt investigation or early surgical intervention. For life-threatening conditions, timely diagnoses and pertinent investigations are essential for appropriate intervention and management.

The response of hypothalamic pituitary adrenal axis is an important defence factor. Cortisol is a principle hormone secreted by adrenal glands and essential to regulate stressful conditions in human body. Its proportional secretion is positively correlated with the degree of illness in hospital- ized medical patients and already shown in research. (3) Also, there are some already known clinical elevated cortisol level situations in literature such as viral infections, psychiatric disorders, psychological stress or exposure. (4-8)

To understand the stress response to a stressful clinical condition in the pathogenesis of acute abdomen, we measured the cortisol levels on ED admission. We hypothesized that higher cortisol levels are related to the laparotomy in patients with acute abdominal pain.

\section{MATERIALS AND METHODS}

This prospective observational crosssectional study was performed in a training and research hospital ED between the dates of November 20, 2013 and December 5, 2013. The ED has 180000 visits annually. Permission for the study was approved by the local ethics committee (302/16.08.2013). Informed consent was obtained from each patient.

The study included consecutive adult patients admitted to the ED with abdominal pain of less than one week's duration. Exclusion criteria have been identified as: 1. Using any form of steroids, 2. Presence of diagnosed cortisol-related disease (Cushing's syndrome or disease, pituitary or ectopic tumour, Pseudo-Cushing's syndrome, Addison's disease, Nelson's syndrome, Sheehan's syndrome), 3. Presence of mental disorder (bulimia nervosa, schizophrenia, major depression), 4. Prior major surgery within the last six months, 5. Pregnancy. 6. Trauma-related abdominal pain.

All subjects were evaluated in the ED triage area and placed in the appropriate 
triage category regardless of the study settings. Demographics, vital signs, and clini$\mathrm{cal}$ and laboratory data were noted. The absolute neutrophil count was divided by the absolute lymphocyte count to calculate the neutrophil-to-lymphocyte ratio (NLR). Patients were examined by an emergency physician and preliminary diagnosis was recorded. Data was collected on an electronic database. Patients were followed up with necessary further investigations as was clinically indicated to ascertain the final diagnosis. Nonspecific abdominal pain (NSAP) was defined as an abdominal pain lasting more than six hours and less than seven days, without fever, leukocytosis, or obvious peritoneal signs and unclear diagnosis after physical exam and baseline investigations.

\section{Cortisol measurements}

When it was decided that a patient should be included in the study, a blood sample for cortisol level testing was drawn. Blood samples were collected with $0.5 \mathrm{ml}$ volume in red top tube via standard venipuncture techniques. If gross hemolysis or visible lipemia were seen, blood sample was repeated. The serum was stored at $-80^{\circ} \mathrm{C}$ until assayed. Serum cortisol levels were measured with an immunochemistry analyser (XP, Siemens Healthcare Diagnostics, Erlangen, Germany) using a solid-phase competitive method.

\section{Outcome measurements}

The primary end-point was defined as differences in serum cortisol levels between surgical and non-surgical treated ED patients with acute abdominal pain. The secondary objective was to compare the accuracy of cortisol with defined complete blood count (CBC) parameters [white blood cell (WBC) count, neutrophil count and percentage, NLR] in predicting surgical treatment in those groups.

\section{Statistical analysis}

Findings were analysed with patients categorized in operated and non-operated groups. All calculations were performed using SPSS version 15.0 statistical software package (IBM, Armonk, NY). Descriptive statistics were reported, including mean, standard deviation, and frequency. The Kolmogorov-Smirnov test was used to check the normality assumption. Continuous data was analysed using the unpaired t-test or Mann-Whitney U test, depending on whether the data was normally distrib- uted. Differences in medians were computed with $95 \%$ confidence intervals (CIs). The $\mathrm{p}$ values for comparisons of categorical variables were generated by the chi-square test. If the relationship between numerical variables did not provide a parametric test condition, Spearman correlation was performed. Also, cut off values were calculated, and ROC curve analysis performed. Area under the receiver operating characteristic curve (AUC) was used to quantify prognostic accuracy. All the statistical analysis was done with $95 \%$ CIs and if the $\mathrm{p}$ value was 0.05 or lower, the result was considered as statistically significant.

\section{RESULTS}

A total of 203 consecutive patients were assessed for eligibility; 92 were excluded for the reasons described in figure 1, and 111 subjects were enrolled in the study. Of them, $53(47.7 \%)$ were female and the median age was 45 (IQR 31-59.5). The baseline characteristics of study participants are presented in Table 1. Three most frequent diagnoses were NSAP (36.6\%), acute appendicitis (9.9\%) and dyspeptic

acute appendicitis (9 patients), acute cholecystitis (4 patients), bowel obstruction (3 patients), perforated viscus (2 patients), ectopic pregnancy-ovarian cyst rupture (2 patients), upper GI (gastrointestinal) bleeding (1 patient), and ruptured abdominal aortic aneurysm (1 patient). All patients' median serum cortisol level was $16.63 \mu \mathrm{g} / \mathrm{dl}$ (IQR: 9.65 to $23.92 \mu \mathrm{g} / \mathrm{dl}$ ). Performance characteristics of laboratory parameters in surgically and non-surgically treated patients are presented in Table 2.

The median cortisol level was $23.13 \mu \mathrm{g} / \mathrm{dl}$ (IQR: $22.5-50.2 \mu \mathrm{g} / \mathrm{dl}$ ) in surgically treated patients and $13.94 \mu \mathrm{g} / \mathrm{dl}$ (IQR: 13.7-29.7 $\mu \mathrm{g} / \mathrm{dl})$ in non-surgically treated patients $(p<0.001)$. Additionally when evaluated defined CBC parameters separately; the medians were found in surgically treated patients and in non-surgically treated patients, 12.95 109L (IQR: 7.43-14.84) and 8.05 109L (IQR: 6.53-10.94) $\quad(\mathrm{p}=0.003)$ for WBC; 10.32 109L (IQR: 5.29-11.94) and 5.37 109L (IQR: 4.01-7.20) $(\mathrm{p}=0.001)$ for neutrophil count; $74.65 \%$ (IQR: 69.1$84.30)$ and $69.04 \%$ (IQR: 58.6-75.6) $(\mathrm{p}=0.007)$ for neutrophil percentage; 5.23 (IQR:3.15-10.13) and 3.43 (IQR:1.89-6.00) $(\mathrm{p}=0.011)$ for NLR, respectively. 
Table 1. The characteristics of study participants

\begin{tabular}{llll}
\hline Characteristic & Non-surgically treated & Surgically treated & p \\
\hline Age, year (median, IQR) & $45(31-60)$ & $37(31-54)$ & 0,482 \\
\hline Gender, male (n, \%) & $49(84.5)$ & $9(15.5)$ & 0,234 \\
\hline Systolic blood pressure, mmHg (median, IQR) & $130(118-140)$ & $122.5(116-135)$ & 0.140 \\
\hline Diastolic blood pressure, mmHg (median, IQR) & $70(65-78)$ & $66(62-76)$ & 0.182 \\
\hline Heart rate, beats in a minute (median, IQR) & $78(72-90)$ & $77.5(74-94)$ & 0.433 \\
\hline Respiratory rate, breaths in a minute (median, IQR) & $14(12-16)$ & $14(13-15)$ & 0.908 \\
\hline Body temperature, oC (median, IQR) & $36.3(36.1-36.6)$ & $36.4(36.1-36.7)$ & 0.342 \\
\hline Duration of pain, hours (median, IQR) & $10(5-48)$ & $12(4-24)$ & 0.595 \\
\hline Hospitalization, yes (n, \%) & $8(26.7)$ & $22(73.3)$ & $<0.001$ \\
\hline IQR
\end{tabular}

IQR, Interquartile Range.

Table 2. Performance characteristics for parameters

\begin{tabular}{|c|c|c|c|}
\hline Parameter & Non-surgically treated & Surgically treated & $\mathbf{p}$ \\
\hline Cortisol, $\mu \mathrm{g} / \mathrm{dl}$ (median, IQR) & $13.94(8.39-21.45)$ & $23.13(18.08-30.01)$ & $<0.001$ \\
\hline WBC, 109/L (median, IQR) & $8.05(6.53-10.94)$ & $12.95(7.43-14.84)$ & 0.003 \\
\hline Neutrophil count, 109/L (median, IQR) & $5.37(4.01-7.20)$ & $10.32(5.29-11.94)$ & 0.001 \\
\hline Neutrophil percentage, \% (median, IQR) & $69.04(58.60-75.60)$ & $74.65(69.10-84.30)$ & 0.007 \\
\hline NLR (median, IQR) & $3.43(1.89-6.00)$ & $5.23(3.15-10.13)$ & 0.011 \\
\hline
\end{tabular}

IQR, Interquartile Range; NLR, Neutrophil to Lymphocyte Ratio; WBC, White Blood Cell Count.

Table 3. Receiver operating characteristic curve of parameters

\begin{tabular}{llllll}
\hline Parameter & Cut-off value & AUC & Sensitivity & Specificity & CI \\
\hline Cortisol & $17.98 \mu \mathrm{g} / \mathrm{dl}$ & 0.750 & $77.3 \%$ & $67.4 \%$ & $0.659-0.827$ \\
\hline WBC & $12.50109 / \mathrm{L}$ & 0.706 & $54.6 \%$ & $87.4 \%$ & $0.611-0.789$ \\
\hline Neutrophil count & $9.99109 / \mathrm{L}$ & 0.721 & $59.1 \%$ & $88.5 \%$ & $0.627-0.803$ \\
\hline Neutrophil ratio & $64.8 \%$ & 0.683 & $90.9 \%$ & $44.8 \%$ & $0.587-0.769$ \\
\hline NLR & 2.87 & 0,677 & $86,4 \%$ & $44,8 \%$ & $0.580-0.763$
\end{tabular}

AUC, Area Under the Curve; CI, Confidence Interval; NLR, Neutrophil to Lymphocyte Ratio; WBC, White Blood Cell Count.

performed to determine the accuracy of WBC, neutrophil count, neutrophil percentage and NLR for predicting surgical disease; the AUCs were calculated as 0.706 (95\% CI, 0.611-0.789), 0.721(95\% CI, $0.627-0.803), 0.683$ (95\% CI, 0.587-0.769) and 0,677 (95\% CI, 0.580-0.763) respectively (table 3 ). The accuracy of cortisol for discriminating surgical disease wasn't inferior to WBC, neutrophil count, neutrophil percentage and NLR.

\section{DISCUSSION}

Abdominal pain is a common chief complaint in EDs. Medical history, physical examination, repeated bedside observations and imaging studies are the most important factors for differential diagnosis of acute abdomen. The key clinical decision is to determine whether the condition is urgent and early surgical intervention is required. The literature shows that, while individual laboratory studies taken in isolation rarely determine a diagnosis, the results of laboratory studies do frequently affect tenancy or treatment in the ED. Cortisol is a surrogate for direct assessment of cytokine generation, which is triggered by most forms of inflammation, infection, and tissue injury. It can be valuable tool in diagnostic assistance, confirming the existence of inflammation, assessing severity, and monitoring the response to treatment and the development of complications. In the present hypothesis-generating study, we demonstrated that elevated serum cortisol levels are associated with increased risk of surgical disease in adult ED patients with non-traumatic acute abdominal pain. This study has two main findings. First, patients in surgically treated group had higher serum cortisol levels. Second, the accu- racy of cortisol to detect surgical disease was not inferior to WBC count, neutrophil count, neutrophil percentage and NLR, which have been used almost routinely for acute abdominal pain evaluation.

Clinical applications of serum cortisol in different disease entities have been published. The studies showed that high serum cortisol levels can predict adverse outcome in patients with severe community acquired pneumonia, cardiac event risk in chronic heart failure, survival state in resuscitated cardiopulmonary arrest, mortality risk in chronic haemodialysis, and development of postoperative cognitive dysfunction following cardiac surgery. (915) To our knowledge, this is the first study to investigate the association between cortisol levels and surgical abdominal pain. It's an important problem to estimate surgical disease in patients with abdominal pain, especially at the early stage of 
admission and we found that cortisol levels were significantly higher in surgically treated patients. In our opinion, it is likely that the stress response to pain represents an exclusive trigger for surgical abdomen occurrence; however, recent findings indicate that interdependent mechanisms, such as inflammatory and stress responses, through interaction effects, may be involved in the pain pathogenesis. $(16,17)$ Many diagnostic tests have been evaluated in research to make differential diagnosis and identify emergent conditions in ED patients with acute abdominal pain. The most common laboratory tests ordered in the $\mathrm{ED}$ are $\mathrm{CBC}$ (34.1\%), blood urea nitrogen (BUN/creatinine) (20.1\%), electrolytes (19.1\%), cardiac enzymes (19\%), and liver function tests (11.5\%). (18) Traditionally, $\mathrm{CBC}$ is the most widely used and guided test in abdominal pain evaluation. As emphasized in many studies in the literature, Abbas et al. reported that important factors which predict acute surgical pathology was found related raised WBC, tachycardia, guarding and vomiting. (19) However, in one study, $25 \%$ of the patients with an elevated WBC did not have different results than those with normal WBC. (20) Parker et al. examined the utility of the $\mathrm{WBC}$ and liver function tests in elderly ED patients with acute abdominal pain and demonstrated that the tests do not distinguish surgical from non-surgical disease. (21) NLR is a predictor of acute inflammation and was recently studied in many clinical conditions for the diagnosis or prognosis. In literature, there are studies that report NLR predict surgical abdomen and also there are studies reporting the opposite. (22-24) In the present study, defined CBC parameters, NLR and cortisol levels were significantly higher in surgically treated patients. Additionally, the accuracy of serum cortisol level to distinguishing surgical from non-surgical disease in acute abdominal pain was not inferior to defined CBC parameters and NLR.

Important limitations must be stated. Our study has been performed with a small number of patients and in a single centre. The collected information doesn't re- flect the prevalence of general population. Further studies should be conducted with larger groups. We could not appropriately reach sample size of different diagnostic subgroups and we could not determine their cortisol levels changes (i.e., acute appendicitis, acute cholecystitis, bowel obstruction). Only a single cortisol level was measured on admission in each patient's hospital course, so we didn't know any information about cortisol level peaks and treatment response. Finally, in some conditions, treatment alternatives may be very diversely based on facilities and capabilities of the institutions (operation or observation).

In conclusion, serum cortisol levels are higher in surgically treated patients. High serum cortisol levels may indicate surgical disease at the early stage on admission in ED patients with non-traumatic acute abdominal pain and may be used as a marker in the prediction of surgical disease.

\section{REFERENCES}

1. National Hospital Ambulatory Medical Care Survey: 2010 Emergency Department Summary Tables (cited 2018 August 15). Available from: https://www.cdc.gov/nchs/data/ahcd/nhamcs_emergency/2010_ed_web_tables.pdf

2. Nagurney JT, Brown DF, Chang Y, Sane S, Wang AC, Weiner JB. Use of diagnostic testing in the emergency department for patients presenting with non-traumatic abdominal pain. J Emerg Med 2003;25(4):363-71.

3. Widmer IE, Puder JJ, König C, Pargger H, Zerkowski HR, Girard J, et al. Cortisol response in relation to the severity of stress and illness. J Clin Endocrinol Metab 2005;90:4579-86.

4. Beishuizen A, Thijs LG, Verries I. Patterns of corticosteroid binding globulin and free cortisol index during septic shock and multi trauma. Intensive Care Med 2001;27:1584-91.

5. Lovallo WR, Farag NH, Vincent AS, Thomas TL, Wilson MF. Cortisol responses to mental stress, exercise, and meals following caffeine intake in men and women. Pharmacol Biochem Behav 2006;83:441-7.

6. King A, Munisamy G, de Wit H, Lin S. Attenuated cortisol response to alcohol in heavy social drinkers. Int J Psychophysiol 2006;59:203-9.

7. Coiro V, Capretti L, Volpi R, Davoli C, Marcato A, Cavazzini U, et al. Stimulation of ACTH/cortisol by intravenously infused substance $\mathrm{P}$ in normal men: inhibition by sodium valproate. Neuroendocrinology 1992;56:459-63.

8. Vaughan GM, Becker RA, Allen JP, Goodwin CW Jr, Pruitt BA Jr, Mason AD Jr. Cortisol and corticotrophin in burned patients. J Trauma 1982;22:263-73.

9. Salluh JI, Shinotsuka CR, Soares M, Bozza FA, Lapa e Silva JR, Tura BR, et al. Cortisol levels and adrenal response in severe community-acquired pneumonia: A systematic review of the literature. J Crit Care 2010;25:541.e1-8.

10. Remmelts HH, Meijvis SC, Kovaleva A, Biesma DH, Rijkers GT, Heijligenberg R. Changes in serum cortisol levels during community-acquired pneumonia: the influence of dexamethasone. Respir Med 2012;106:905-8.

11. Yamaji M, Tsutamoto T, Kawahara C, Nishiyama K, Yamamoto T, Fujii M, et al. Serum cortisol as a useful predictor of cardiac events in patients with chronic heart failure: the impact of oxidative stress. Circ Heart Fail 2009;2:608-15.

12. Ito T, Saitoh D, Takasu A, Kiyozumi T, Sakamoto T, Okada Y. Serum cortisol as a predictive marker of the outcome in patients resuscitated after cardiopulmonary arrest. Resuscitation 2004;62(1):55-60.

13. Gracia-Iguacel C, González-Parra E, Egido J, Lindholm B, Mahillo I, Carrero JJ, et al. Cortisol levels are associated with mortality risk in hemodialysis patients. Clin Nephrol 2014;82:247-56.

14. Glumac S, Kardum G, Karanović N. A Prospective Cohort Evaluation of the Cortisol Response to Cardiac Surgery with Occurrence of Early Postoperative Cognitive Decline. Med Sci Monit 2018 Feb 17;24:977-86.

15. Dikme O, Dikme O. Serum Cortisol as a Predictor of Major Adverse Pulmonary Event in Emergency Department Acutely Dyspneic Patients. Emerg Med Int Volume 2018, Article ID 1758643.

16. Strittmatter M, Bianchi O, Ostertag D, Grauer M, Paulus C, Fischer C, et al. Altered function of the hypothalamic-pituitary-adrenal axis in patients with acute, chronic and episodic pain. Schmerz 2005;19(2):109-16.

17. Tennant F. The Physiologic Effects of Pain on the Endocrine System. Pain Ther 2013;2(2):75-86. 
18. Pitts SR, Niska RW, Xu J, Burt CW.National Hospital Ambulatory Medical Care Survey 2006 emergency department summary. Natl Health Stat Report 2008;7:1-38.

19. Abbas SM, Smithers T, Truter E. What clinical and laboratory parameters determine significant intraabdominal pathology for patients assessed in hospital with acute abdominal pain? World J Emerg Surg 2007;25;2:26.

20.Lukens TW, Emerman C, Effron D. The natural history and clinical findings in undifferentiated abdominal pain. Ann Emerg Med 1993;22:690-6.

21. Parker JS, Vukov LF, Wollan PC. Abdominal pain in the elderly: use of temperature and laboratory testing to screen for surgical disease. Fam Med 1996;28:193-7.

22. Markar SR, Karthikesalingam A, Falzon A, Kan Y. The diagnostic value of neutrophil: lymphocyte ratio in adults with suspected acute appendicitis. Acta Chir Belg 2010;110(5):543-7.

23. Cigsar G, Yildirim AC, Anuk T, Guzel H, Gunal E, Gulkan S, et al. Neutrophil to Lymphocyte Ratio on Appendectomy of Geriatric and Nongeriatric Patients. J Invest Surg 2017;30(5):285-90.

24. Tanrikulu Y, Sen Tanrikulu C, Sabuncuoglu MZ, Kokturk F, Temi V, Bicakci E. Is the neutrophil-to-lymphocyte ratio a potential diagnostic marker for peptic ulcer perforation? A retrospective cohort study. Am J Emerg Med 2016;34(3):403-6. 